\title{
Covid-19 Epidemic Control Strategy Using Risk Analysis and Decision Making Tools
}

\author{
Mahfoud CHAFAl, Abdelkader ZITOUNI, Hamid BENTARZI* \\ Laboratory Signals and Systems (LSS), IGEE, University M'hamed BOUGARA Boumerdes, \\ Algeria. \\ *Corresponding e-mail: Iss@univ-boumerdes.dz
}

\begin{abstract}
Mitigation of the major risk caused by the Coronavirus epidemic must be approached in a scientific manner by using methods and tools of risk analysis and management. In order to develop an effective strategy, emergency plan based on these methods for minimizing human losses whatever the scenario that may arise in the future may be established.
\end{abstract}

Keywords: Covid-19 Epidemic, risk analysis and management, Decision making.

\section{INTRODUCTION}

Major risk management from an intervention perspective is defined as:"an organized effort to identify, assess and reduce risks wherever possible" as illustrated in Fig.1. It is fundamentally based on:

- Identification of hazards (viruses), their sources and the biological risks involved,

- Quantitative evaluation: statistical trends, risk index and analytical assessment,

- Reduction of risk to an acceptable level or its total elimination.

However, it is a regular, iterative process of continuous improvement, coordinated and integrated throughout the organization that allows for the identification, control and evaluation of risks [1].

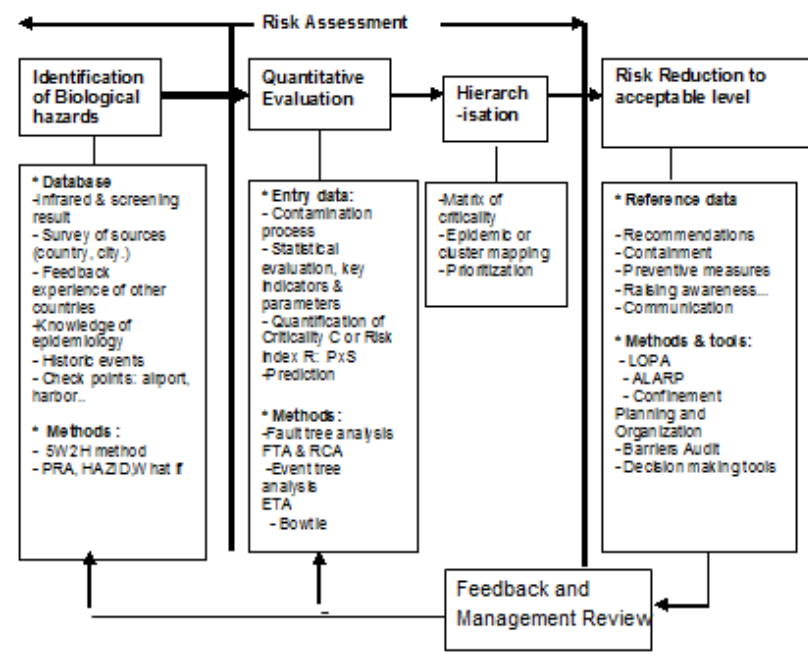

Fig. 1 Global procedure.

\section{IDENTIFICATION OF SOURCES OF BIOLOGICAL HAZARDS}

It is a process of systematically collecting medical information and data that will first be used to identify all the risks to which medical workers, the public and the environment are exposed and then analyzing them. This data collection is based on:

- The identification of the countries of high risk (>stage 3) and source of the danger of the virus (via nations), the outbreak of the epidemic area, the clusters and the points of contact or passage between the infected areas and the areas to be protected such as airports, ports, road transport.

- Systems for medium and targeted screening, testing and detection of symptomatic and asymptomatic infected persons using infrared camera, biological PCR and Scanner imaging system. Once detected, the potentially sick persons are isolated or put in quarantine for further treatment.

- The 5W2H (Why-What -Where-When-WhoHow-How much ?) method, investigation and data collection methods involving a virtually exhaustive list of information to identify infected suspects and those who were in contact, in order to identify and locate people who were in contact with the person transmitting the infection. In fact, tracing patient interactions can be traced back to patient zero in each household,

- Feedback experience from countries such as: China, Italy, Germany, France, South Korea (rapid on-site screening test). 
Table 1 Table of Preliminary Risk Analysis (PRA)

\begin{tabular}{|c|c|c|c|c|c|c|c|}
\hline Hazard & Source & $\begin{array}{l}\text { dangerous } \\
\text { Situation }\end{array}$ & $\begin{array}{l}\text { Trigger } \\
\text { event }\end{array}$ & $\begin{array}{l}\text { Hazardous } \\
\text { event }\end{array}$ & Consequence & $\begin{array}{l}\text { Index } \\
\mathbf{R}\end{array}$ & $\begin{array}{l}\text { preventive } \\
\text { Mesures }\end{array}$ \\
\hline \multirow[t]{3}{*}{$\begin{array}{l}\text { Corona } \\
\text { Virus }\end{array}$} & $\begin{array}{l}\text { Contaminated } \\
\text { peoples } \\
\text { coming from: } \\
\text { France and } \\
\text { foreigners }\end{array}$ & $\begin{array}{l}\text { Virus Spores } \\
\text { on Surfaces, } \\
\text { Object, } \\
\text { Handle, } \\
\text { money }\end{array}$ & $\begin{array}{l}\text { Hand } \\
\text { Contact }\end{array}$ & Infection & $\begin{array}{l}\text { Covid-19 \& } \\
\text { Propagation }\end{array}$ & IV & $\begin{array}{l}\text { - Border Closure } \\
\text { (CCP) } \\
\text {-Stopping Air and } \\
\text { Naval Transport }\end{array}$ \\
\hline & & $\begin{array}{l}\text { Spores de } \\
\text { virus in the } \\
\text { air }\end{array}$ & $\begin{array}{l}\text { Airborne } \\
\text { droplet } \\
\text { projection } \\
\text { (cough, } \\
\text { sneeze } \\
\text { talking...) } \\
\text {-Air flow } \\
\text { Air } \\
\text { conditioner }\end{array}$ & Infection & $\begin{array}{l}\text { Covid-19 \& } \\
\text { Propagation }\end{array}$ & IV & $\begin{array}{l}\text { - Screening \& Test } \\
\text { - Isolation- } \\
\text { quarantine }\end{array}$ \\
\hline & $\begin{array}{l}\text { Contaminated } \\
\text { persons from } \\
\text { Outbreak } \\
\text { (Blida } \\
\text { Algiers) }\end{array}$ & $\begin{array}{l}\text { Virus on } \\
\text { Surfaces, } \\
\text { Object, } \\
\text { Handle, } \\
\text { money.. }\end{array}$ & $\begin{array}{l}\text { Hand } \\
\text { contact } \\
\text { and } \\
\text { airborne } \\
\text { route }\end{array}$ & Infection & Covid-19 & IV & Total lockdown \\
\hline
\end{tabular}

-Others: incubation time and new symptoms (loss of smell and taste), proportion of transmission that appears to occur before the onset of symptoms, proportion of the population that is ageing or has a chronic disease...

All these must be organized in a continuously improved and updated database using tabular analysis methods such preliminary risk analysis HAZID, PRA as shown in table I.

\section{QUANTITATIVE AND ANALYSIS}

EVALUATION

A. Evaluation:

Evaluation consists of:

- Statistical evaluation : The collection of information and obtained data, which must be reliable, is represented in tabular, graphical (histogram) or trend form showing the evolution of the number of confirmed infected cases, suspected cases, deaths and recovered cases.

Figure 2-a shows that the evolution of the number of confirmed cases is accelerated since 22nd June onwards, this rate is relatively small compared to that of other countries. The increase in the number of newly infected people is due to the noncompliance of the citizens with the barrier measures and social distancing. We cite the grouping of people in markets, closed post PTT stations, during traditional marriage celebrations or funeral of death. The declared clusters are located in large cities with high population concentration (Setif, Algiers, Blida, Oran ...) in Algeria.

It is well shown that the mortality rate has substantially decreased from $15 \%$ in April to $6 \%$ in July and thus is near the normality [2]. More operational data are needed for better explanation and interpretation such as population with a high rate of chronic disease, elderly or delayed medical management...) as shown in Fig.2-b.

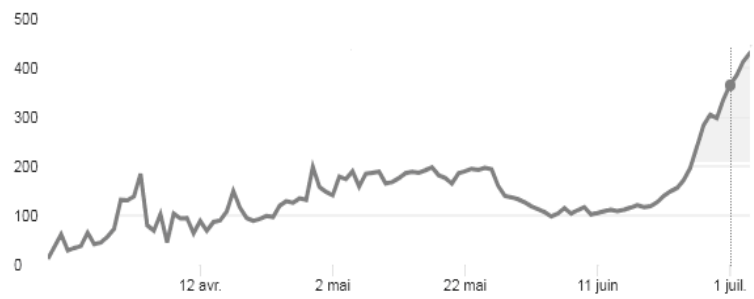

a-Number of confirmed Coronavirus cases

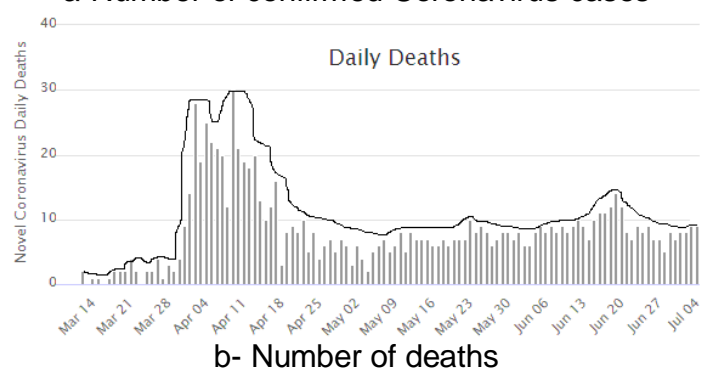

Fig.2 Graphic evolution and trend from the collected data.

This also allows monitoring and observation of the peak, the moment of stability and inversion of the curve and even making extrapolations and predictions. 
The effectiveness of ratio type indicators are those that allow comparison with a reference, of itself and periodically, or a state of evolution of countries such as China, Germany. Algeria:

Example of evaluation key indicators in

- Number of people healed/Total number of people confirmed positive $=11$ $492 / 15941=72 \%$,

- Number of deceased persons/Total number of persons confirmed positive $=$ $952 / 15941=6 \%$,

- Incidence ratio: 38/100000.

- Recovered patients (under Chloroquine treatment): 12094

\section{- Patient in intensive care around: 50}

The therapeutic protocol based on Chloroquine, a drug commonly used against malaria, has shown its "almost total" effectiveness against the Coronavirus in Algeria. The recovery matrix exceeds the bar of 12,000 cases and therefore a cure rate of the coronavirus "exceeds $90 \%$ " of cases.

Thus, the value of applying the action plan, the treatment protocol or making continuous improvement decisions can be verified. In fact, a dashboard based on relevant indicators can be built for medical commission to guide their decisions and actions in order to achieve the designated objectives.

- Risk assessment : For each of the consequences of the propagation scenario studied, the severity $S$, the frequency/probability of occurrence $P$ and the kinetics of the event will be assessed in an increasing manner. The combination of the two parameters of $P$ and $S$ gives the index or level of risk of the feared corona virus infection event.

Severity $S$ (consequence) is expressed in terms of:

- Human losses (case-fatality rate), patients, medical staff, intensive care charge. In this context, for the infected patient severity may include the vulnerability of the targets (e.g. people with, chronically ill, ageing). Furthermore, it may include the incidence of loss of patients on the other hidden medical domains (cancer, urgent surgery..).

- Economic and social impact: economic recession, closure of businesses leading to large-scale unemployment, poverty and famine social, psychological and mental consequences.
The quantification of the probability $\mathrm{P}$ is then carried out by multiplying the values of the probability of occurrence ( $\mathrm{Po}$ equivalent to that of emission), by the probability or frequency of exposure (Pex) and the probability of avoidance (Pev):

$$
\mathrm{P}=\mathrm{Po}{ }^{*} \mathrm{Pex}{ }^{*} \mathrm{Pev}
$$

The replication rate $(>3)$ corresponds to the propagation speed and thus the kinetics of the event. In this case it is estimated upwards.

The Ranking of risk index is required and is a means used by those in charge of risk management and/or to develop a strategy through a good management of priorities. The assignment of probabilities in each phase of the process will highlight the factor of importance of each CoronaVirus control operation.

If the risk analysis is carried out properly, it will lead to a set of recommendations that reflect the priority, i.e. a list of extreme measures that should be taken to eliminate or limit the risks or mitigate the CoronaVirus epidemic is shown in Table I.

\section{B. Analysis : It is based on:}

1) Methods of searching for the causes of undesired 'epidemic' effects (Fault tree or RCA):

It is a deductive method where one starts from the presumed undesired effect of an epidemic and looks for all the causes and combinations of causes that may lead to this effect. The relationship is modeled in a treelike structure with logical AND/OR gate. The numeric fault tree is used for determining the probability of the top event.

The search for causes is pushed to reach the root cause (RCA) as illustrated Fig.3.This method is at the origin of the design and strategic implementation of prevention barriers.

\section{Application: COVID-19 disease occurrence process \\ - Transmission modes}

The virus is primarily spread between people during close contact, most often via small droplets produced by coughing, sneezing, and talking. The droplets usually fall to the ground or onto surfaces currently in contact with people or can land in the mouths or noses of people who are nearby or possibly be inhaled into the lungs [8] (See Fig. 3).

The possibility of airborne transmission of Coronavirus was recently announced by the $\mathrm{WHO}$ and is expected to be responsible for the acceleration of the epidemic worldwide. 


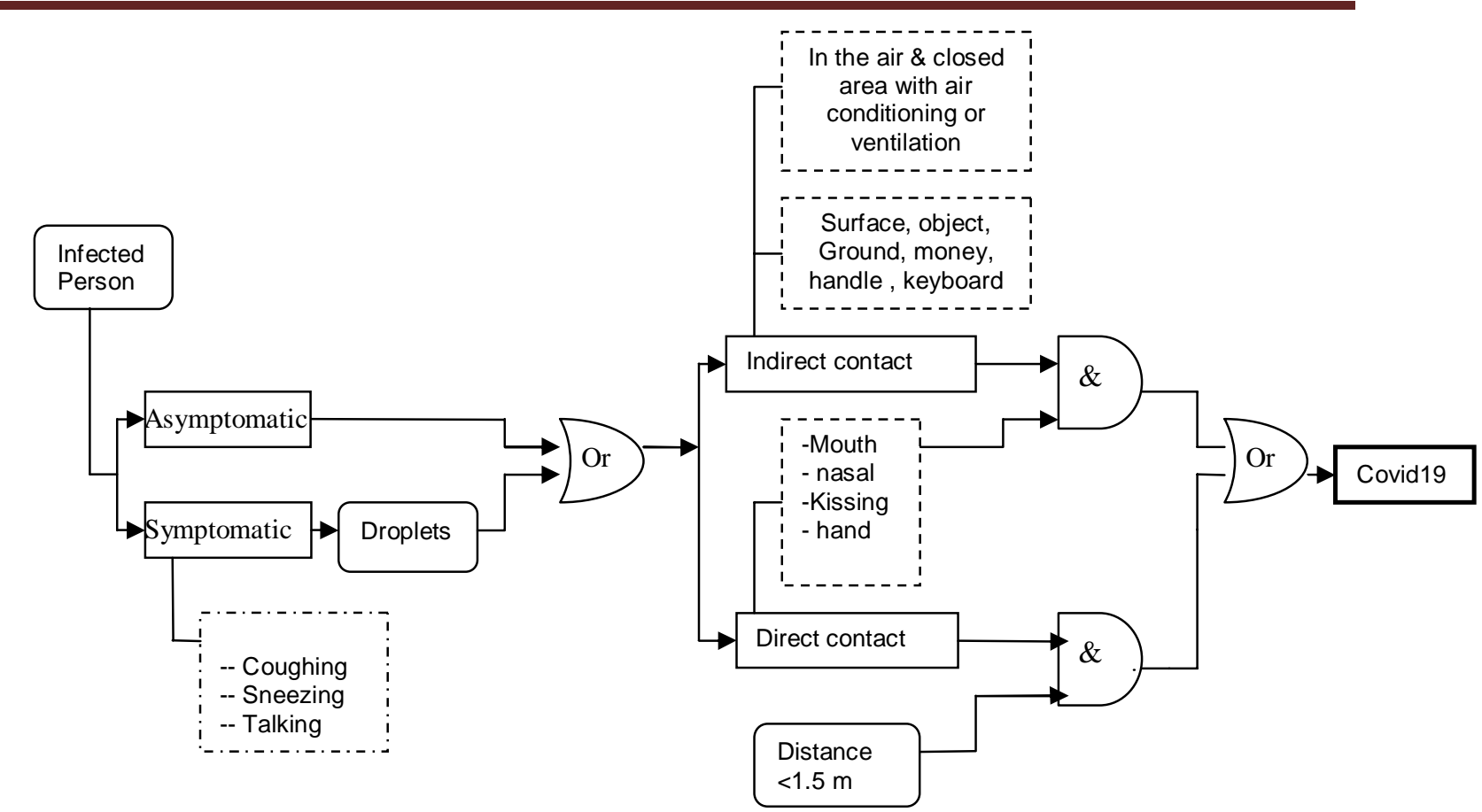

Fig.3 Transmission paths

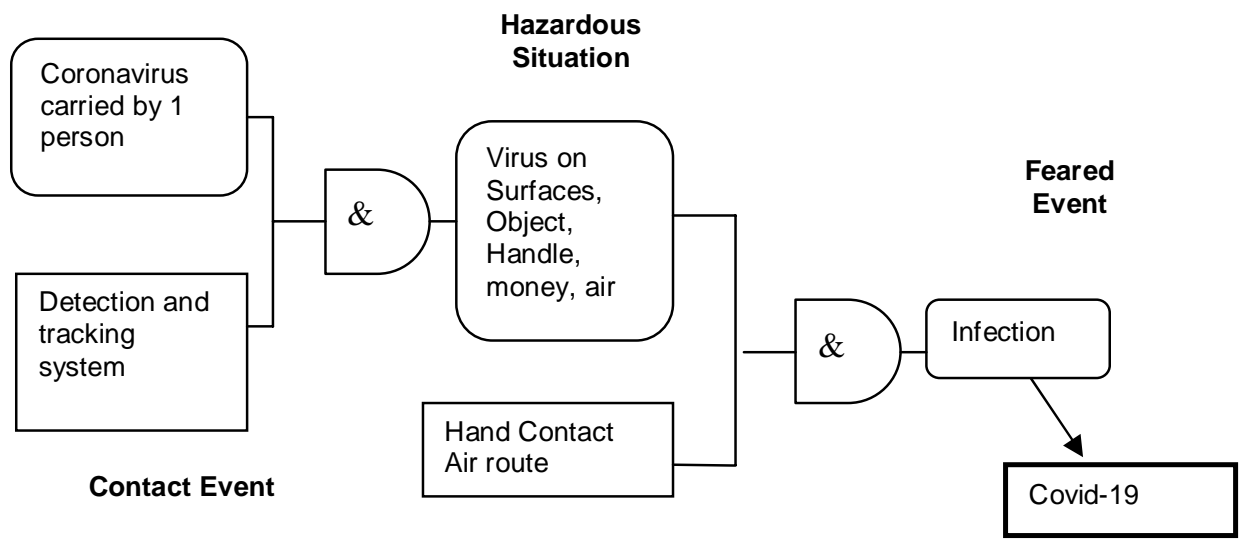

Priming Event

Consequence

Fig.4 Process of occurrence COVID-19

\section{- Modelling the Process of occurrence COVID-19}

The dangerous situation consists of the presence of the person and the biological hazard (Coronavirus) carried by a person. The occurrence of a triggering or initiating event (through the different transmission paths) gives rise to the phenomenon or event feared of infection and as a consequence the occurrence of Corvid-19 disease as shown in Fig. 4 [3].
2) Methods of identifying the consequences and possible scenarios of a situation of epidemic risk (protection and mitigation): consequence tree or Event tree analysis (ETA).

This inductive method, derived from the decision tree, allows the elaboration and evaluation of sequences of events. Starting from a central dreaded event (CDE), it branches out into branches qualified as unacceptable or acceptable or according to the barriers put in place depending on the outcomes or generated consequences as seen in Fig.5. The resulting consequences are grouped according to their severity and the probability of occurrence. 


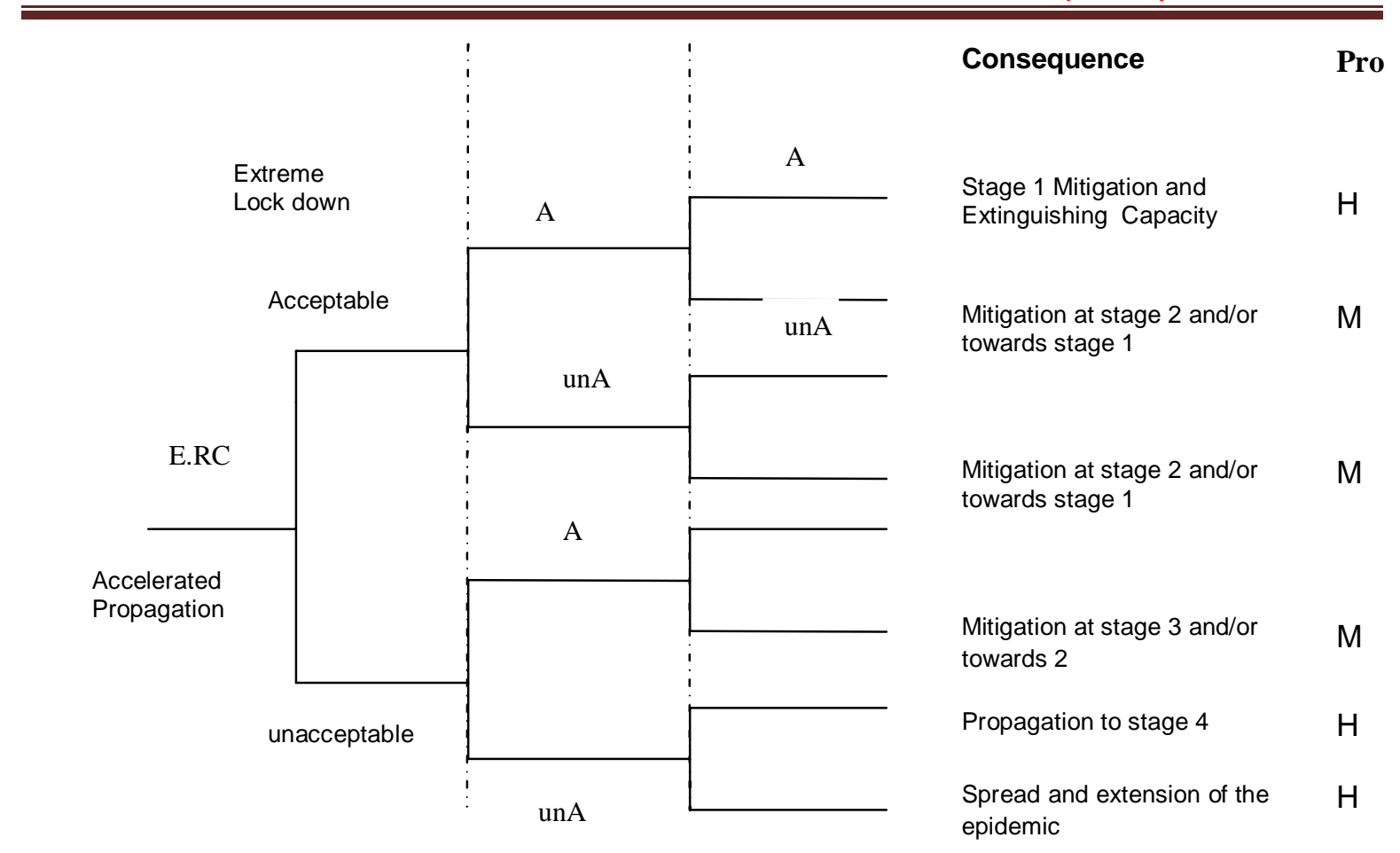

Fig.5 Event tree analysis (Pro: Probability of the outcome $\mathrm{H}=$ High, $\mathrm{M}=$ medium, )

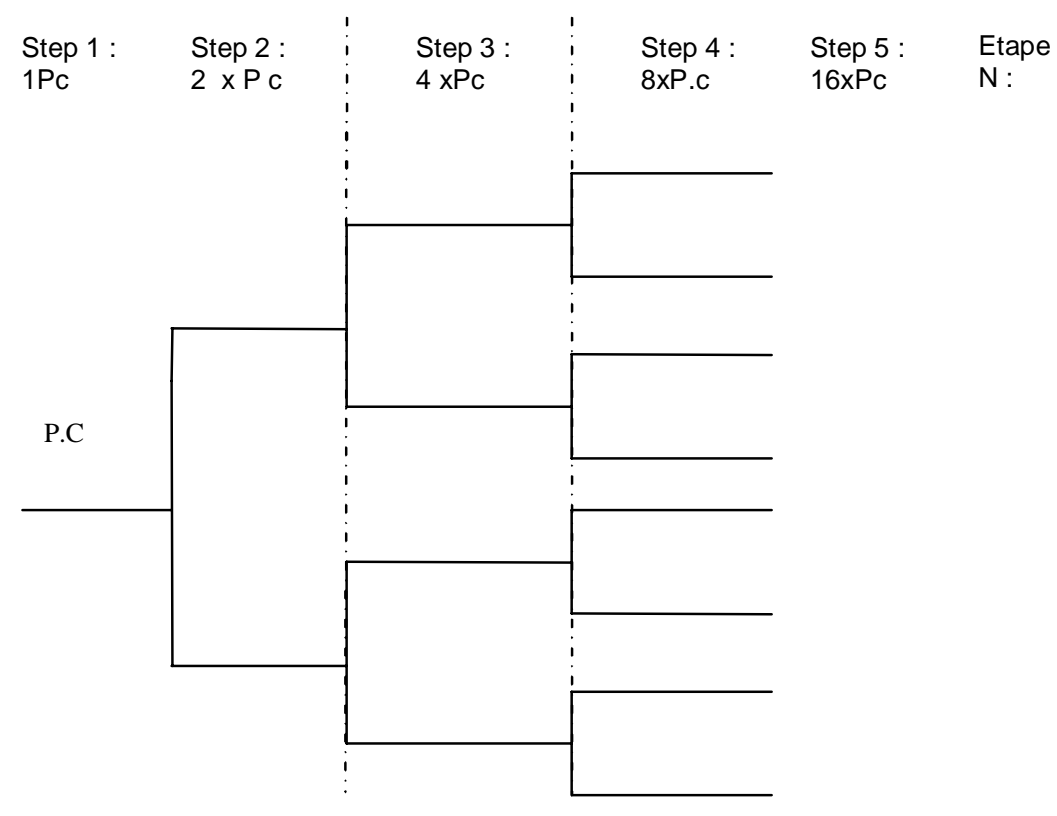

Fig.6 ETA for contamination chain

$$
\mathrm{Npc}=2 \mathrm{Nx} \mathrm{Pc}
$$

This method is at the origin of the strategic implementation of protection and mitigation barriers. Furthermore, the event tree could be used to evaluate the speed of the propagation. In the following example (see Fig.6), if we assume that one infected person (Pc) will infect two peoples at each stage of contact with other people, we will show a very rapid numerical progression of the number of infected people of the order:
Where $\mathrm{N}$ represents the number of contact steps.

In the case where the replica rate is equal to 3 , the propagation will be faster: $\mathrm{Npc}=3 \mathrm{~N}$.

In order to limit or break this chain of progression of Corvid-19, it is imperative to move towards either partial or total confinement with screening according to the established clusters mapping. 


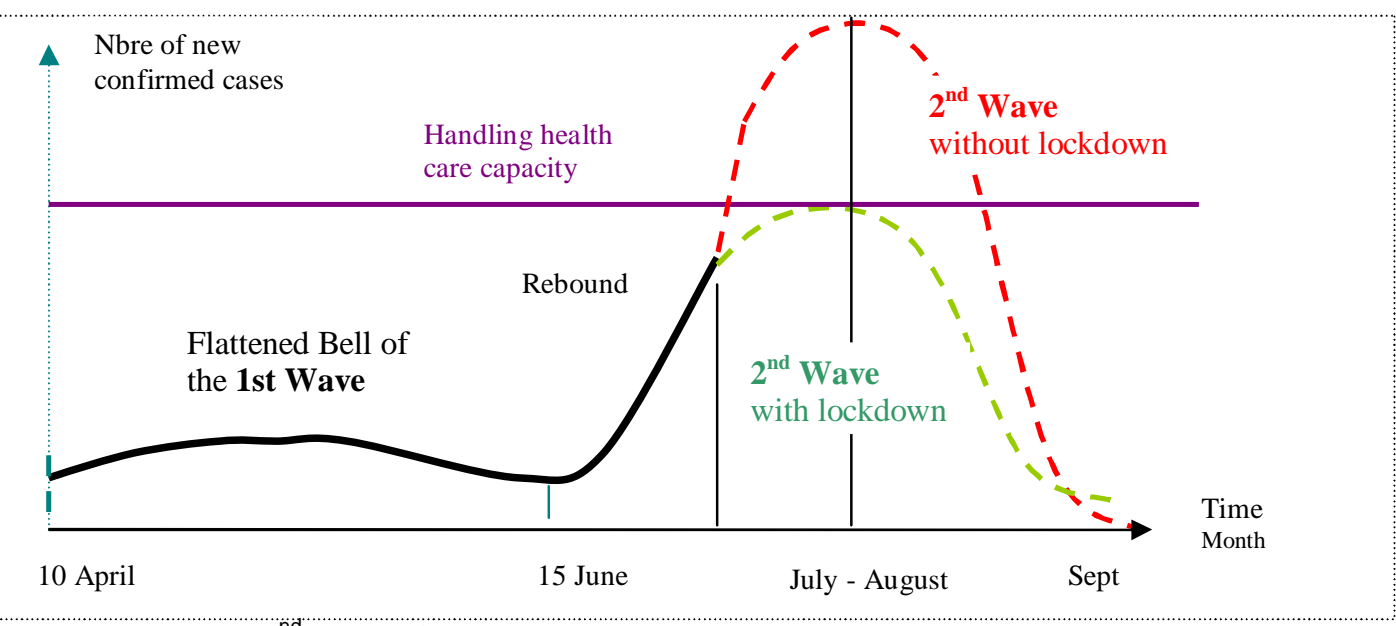

Fig.7 Possible $2^{\text {did }}$ Wave of the epidemic

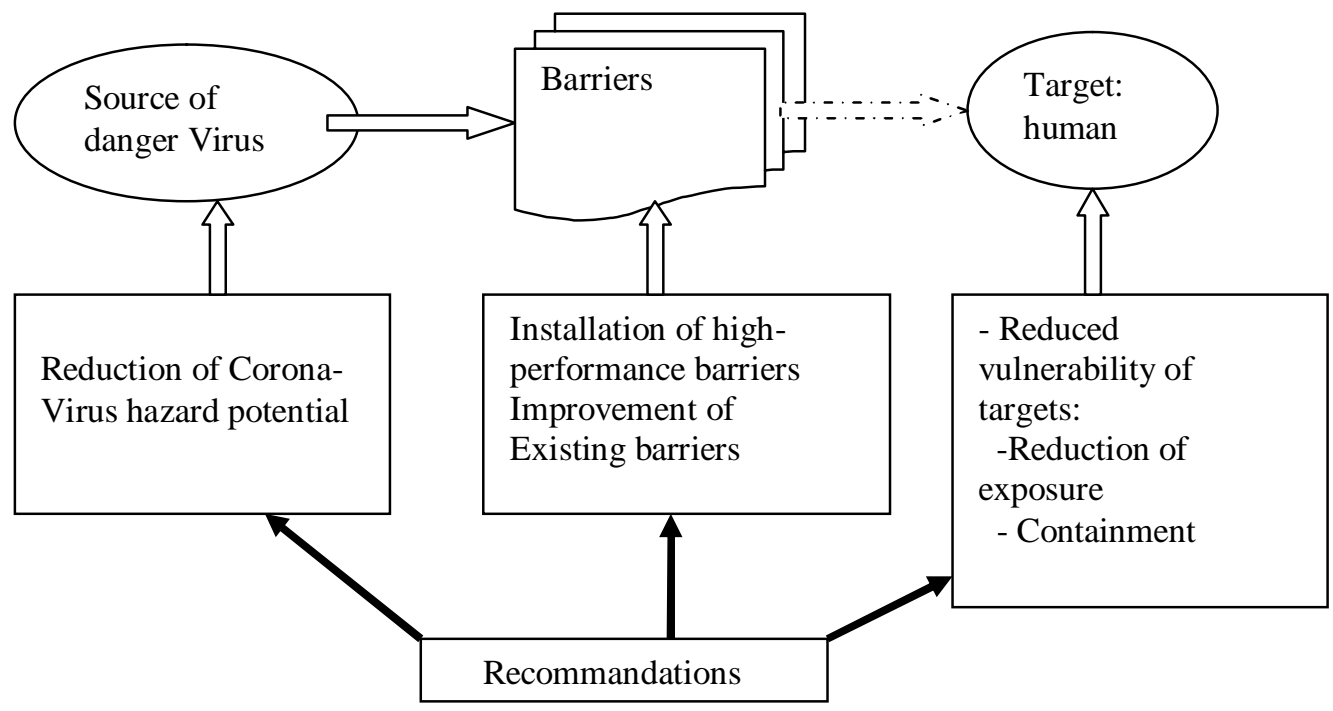

Fig.8 Barriers and recommendations

\section{Prediction of Possible scenario of second wave}

The success of the lockdown is not in itself the end of the epidemic. New outbreaks of contamination and clusters, mostly due to people gathering or found in closed places are becoming more or more frequent, causing the authorities to fear the scenario of a second wave and thus forcing some governments to set up local containment.

After the first one, a second very important wave, as shown in Fig.7, started and would occur possibly in July/August 2020, followed by a progressive extinction with some lighter peaks. This pattern is similar to what was observed with the 1918-1919 pandemic [9].

The "What if" method, which can be used in phase, is a brainstorming carried out by a multidisciplinary group of experts. Questions are asked about the future deviations and experts in the field will answer for further anticipation.

\section{RISK REDUCTION TO AN ACCEPTABLE LEVEL OR ITS ELIMINATION ANALYSIS}

Within the framework of safety and public health studies, safety measures (organizational or technical, prevention, limitation, protection and/or intervention) must be put in place to control risks such as: prevention and protection barriers. See Fig.8.

Barrier performances are evaluated according to the criteria of Effectiveness (capacity to fulfill the safety function assigned to them) and Response Time (time required to fulfill the function adapted to the kinetics of the epidemic propagation phenomenon). The rigor of application through the involvement of the government legal forces (police, military..) is necessary in the case where the recommended measures are not respected. 


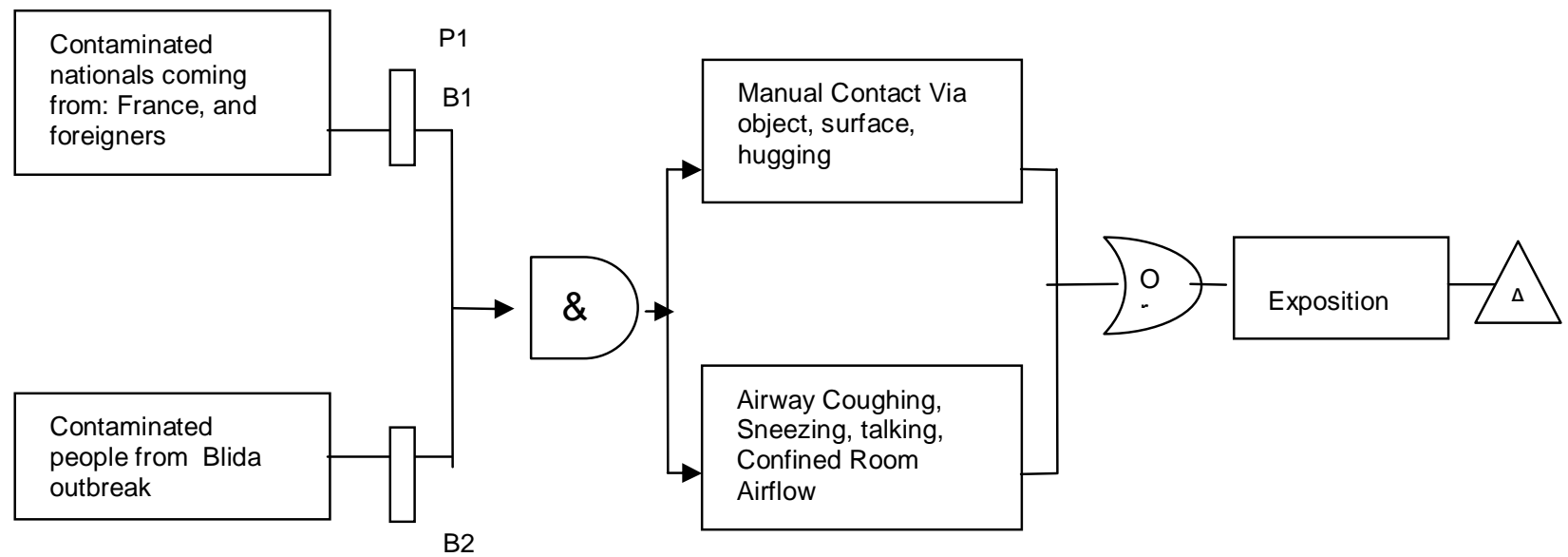

P2

Fig.9 Causal chain of the undesirable effect

"Exposure of people to Coronavirus" [6]

biological hazard could be in case of propagation.

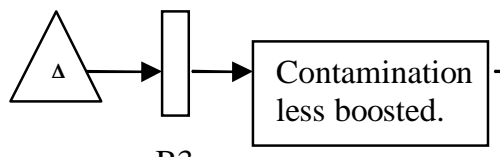

B3

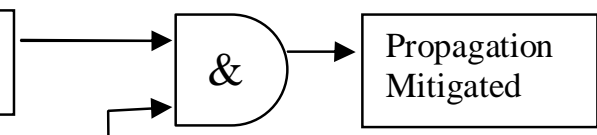

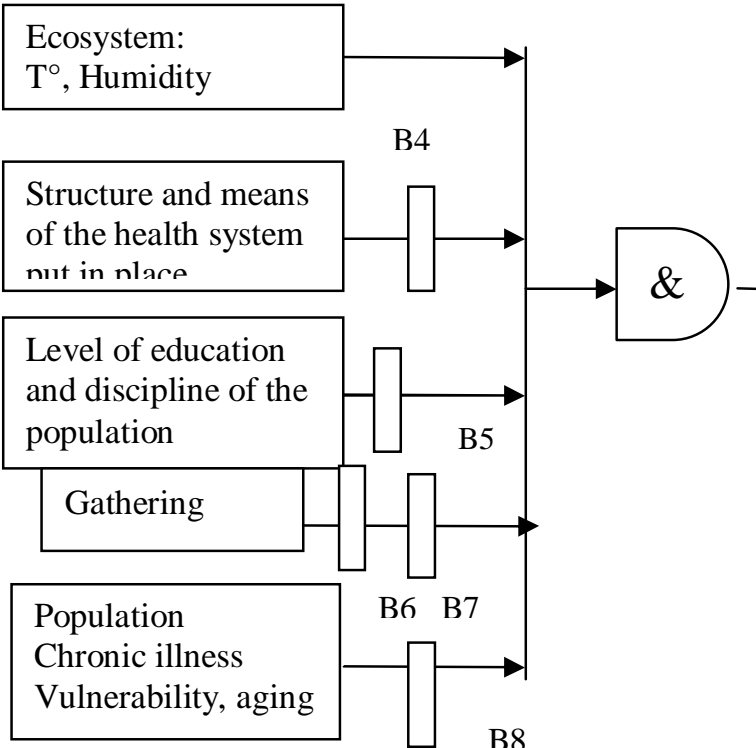

Contribution

Factors

Fig.10 Contamination and contribution factors for the acceleration of the propagation [6]

\section{A. Prevention and Protection or Mitigation}

To combat the risk of epidemic, protective and/or preventive measures must be implemented, which constitute the elements of risk control. Prevention reduces the likelihood of contamination by implementing measures to prevent Covid-19 pathology from occurring. As for protection, it reduces the seriousness, by limiting what the impact of the
These risk reduction or control measures may be human, organizational (procedures, training, communication), or technical [10]. The measures must be taken at the right time, in the right place with an appropriate response time and rigor.

In Figures 10 and 11, cause trees (with causal chain sequences) are presented as well as the implementation of barriers with a 
risk index or priority rating. Management of intensive care requiring artificial respiration and the adoption of a chloroquinezythromycin treatment protocol reduce the degree of severity S.
The risk reduction factor is deduced as the inverse of this probability:

$$
\mathrm{RRF}=1 / \mathrm{PFDavg}
$$

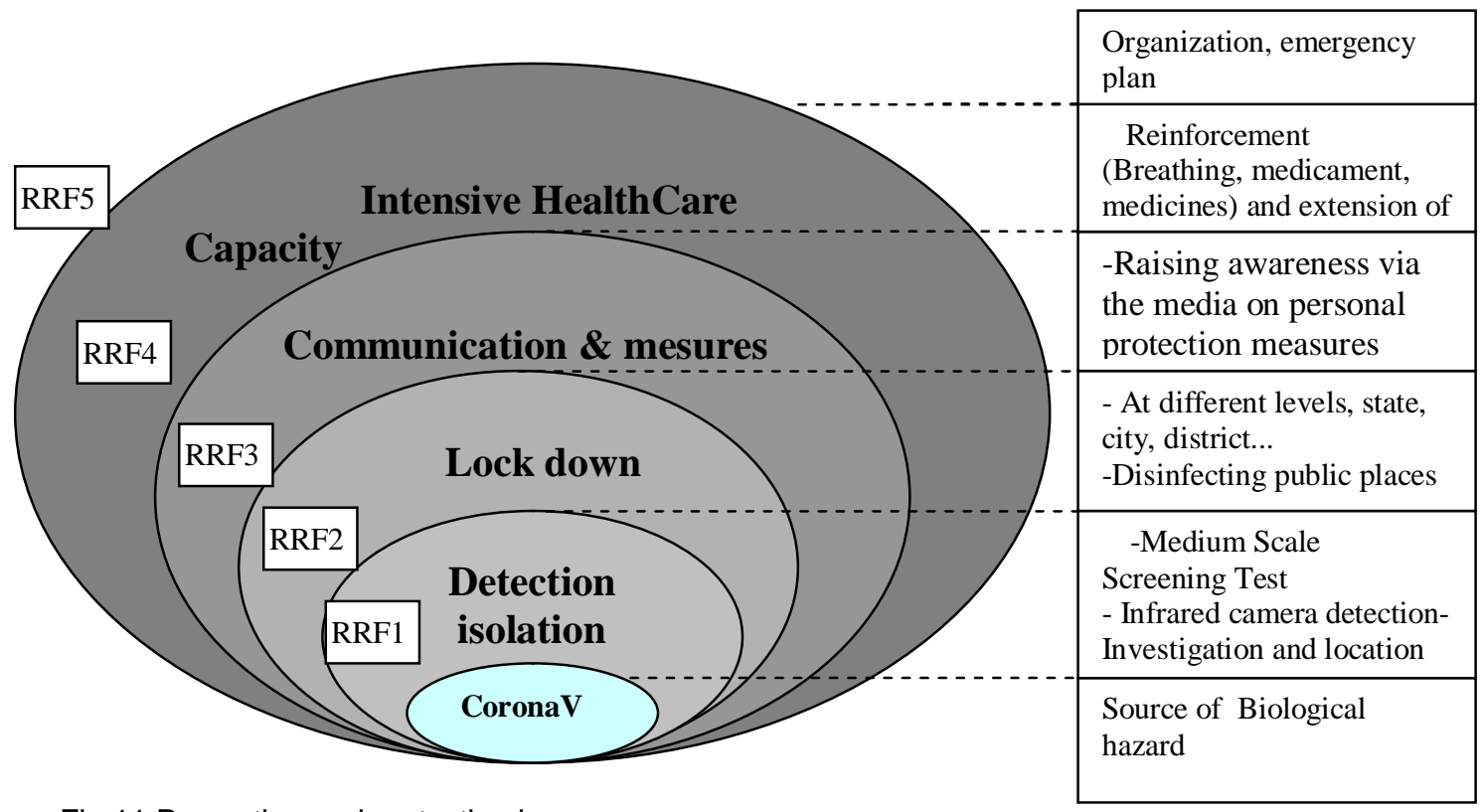

Fig.11 Prevention and protection layers

\section{B. Use of the LOPA (Layer of Protection Analysis) method}

The LOPA method (used in the petrochemical industry) could be applied in this context. It allows assessing the risk reduction by analyzing the contribution of the different layers that encompass all barriers, from the source to the emergency measures in case of propagation. It has the advantage of enhancing the value of all protective layers.

Safety is therefore ensured by layers of protection that begin with the control of infection processes at the source (nationals, tourists, cluster), then extend to the layers of prevention and mitigation of the consequences of the spread of the disease. See Fig. 11.

The 1st layer is of high importance and requires Strategy of screening and testing. We should increase and modulate reliable screening and test of corona virus according the established cluster mapping or localized outbreaks. Biological PCR test is supplemented by the imaging CT scanner that clearly shows lesions of viral aggression.

\section{- Average probability of failure on demand: PFDavg}

This is the average probability that the system will not be able to perform the protection function for which it was designed at the time the request for this function is made.
The overall reduction in the risk of CoronaVirus spread is given as follows:

$$
\mathrm{RRF}_{\mathrm{G}}=\mathrm{RRF} \times \mathrm{RRF} 2 \mathrm{RRF} 3 \mathrm{RRF} 4
$$

If we assign a value of reduction factor $=10$ to each layer then we will have a reduction in the risk of propagation of:

$$
\mathrm{RRF}_{\mathrm{G}}=10^{4}
$$

\section{Use of other risk analysis 1- Bowtie risk analysis method}

The combination of the above cause and consequence tree methods will result in the so-called Bowtie method. This latter will help in the strategic and optimal implementation of both prevention and protection barriers with some quantitative assessment of the importance factor of the barriers.

\section{2- ALARP Zone: "as low as reasonably practicable":}

The risk of applying the chloroquinezythromycine, dexamethasone, remdesivir or Tocilizubab treatments protocol is accepted with the condition that all possible measures to reduce the risk of contraindications (e.g. for cardiac patients) have been respected and implemented. In this case, the impact on the health of a population under treatment, with the advantage that the low cost drug is manufactured locally, is low compared to the expected benefits and in the absence of other 
alternatives and while waiting for specific effective treatment and vaccine .
An example of Gantt planning of is illustrated in the figure 13. In the case the vaccine project, once elaborated it requires more than 10 months for production, packaging and delivery to the various countries.

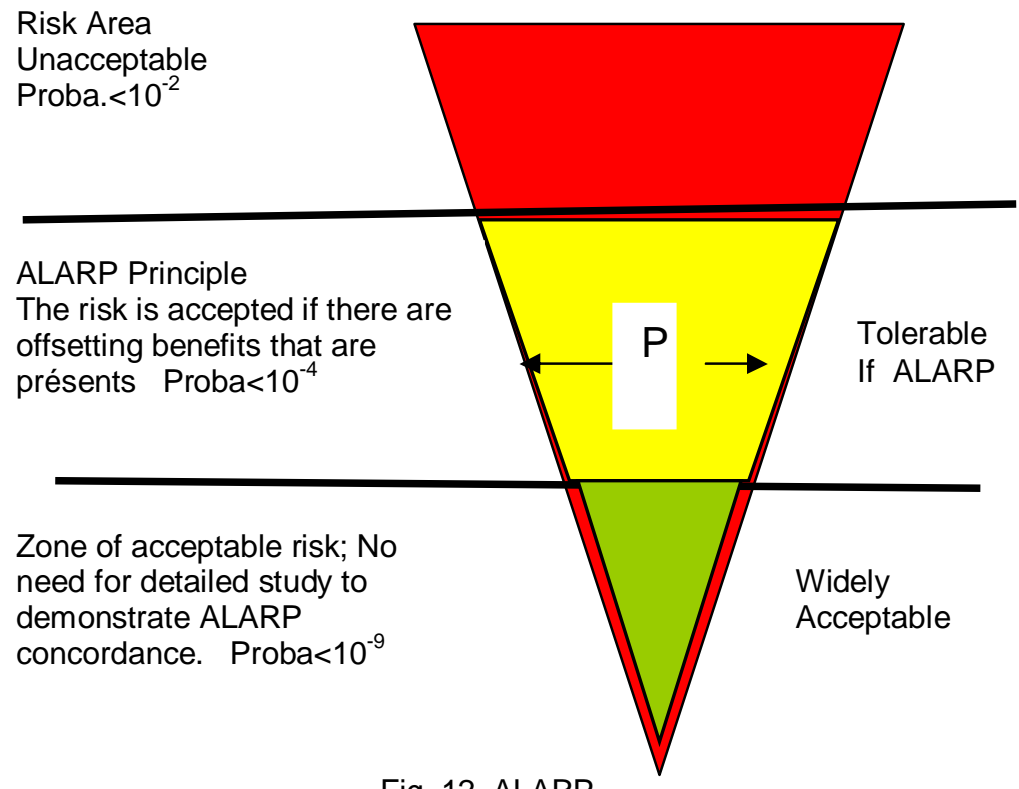

Fig .12 ALARP

\begin{tabular}{|c|c|c|c|c|c|}
\hline \multicolumn{5}{|c|}{ Medical sceening \& Traetement of Covid-19 } & \\
\hline \multicolumn{5}{|c|}{ Prevention - Barrier measures- Lockdowns } & \multirow{3}{*}{ Vaccin } \\
\hline 1st phase Vaccin & $2^{\text {nd }}$ phase Vacc & $3^{\text {rd }}$ phase Vac & Production & Delivery & \\
\hline \multicolumn{3}{|c|}{$\begin{array}{l}\text { Clinical trials } \\
\text {. }\end{array}$} & \multicolumn{2}{|c|}{ Milestone } & \\
\hline July & 2020 & $\longrightarrow$ Dec. & Jan. & 2021 & July \\
\hline
\end{tabular}

Fig.13 Gantt planning

\section{Planning and decision making tools}

The Global risk security GHS Index finds severe weaknesses in country abilities to prevent, detect, and respond to health emergencies; severe gaps in health systems; vulnerabilities to political and socioeconomic [7]. .

Fighting against this Coronavirus epidemic is more than a mega-project which therefore requires a quality management including Tasks such as :

- Optimal planning using tools such as Gantt, PERT, Petri networks to ensure good coordination and scheduling the different activities especially in this phase of uncertainty.
- Methods for rational management of the supply chain, food supplies and basic necessities to contain confinement over time.

- Methods for managing priorities (Pareto) and weight factor (Fussel-Vesely) and decision making tools.

Confinement and the criticality of the health situation-economic growth parameters evolve in opposite directions: when containment decreases, the other two increase. In this context, it will be necessary to seek the best trade-off to achieve the objective of optimization (see Fig. 14).

Targeted reopening is used to safeguard economic and social life. After containment, which appeared to be a beneficial protection, Reopening is the high-risk phase. Slackening, 
recklessness of the population and "noncompliance" with the rules of prevention and protection causes the number of contaminations to rise sharply. In this case, Lockdown-reopening plan or management is

\section{CONCLUSIONS}

Improvements can be obtained through:

- Adequate and additional staffing: concerns intensive care, laboratory Covid-19 test

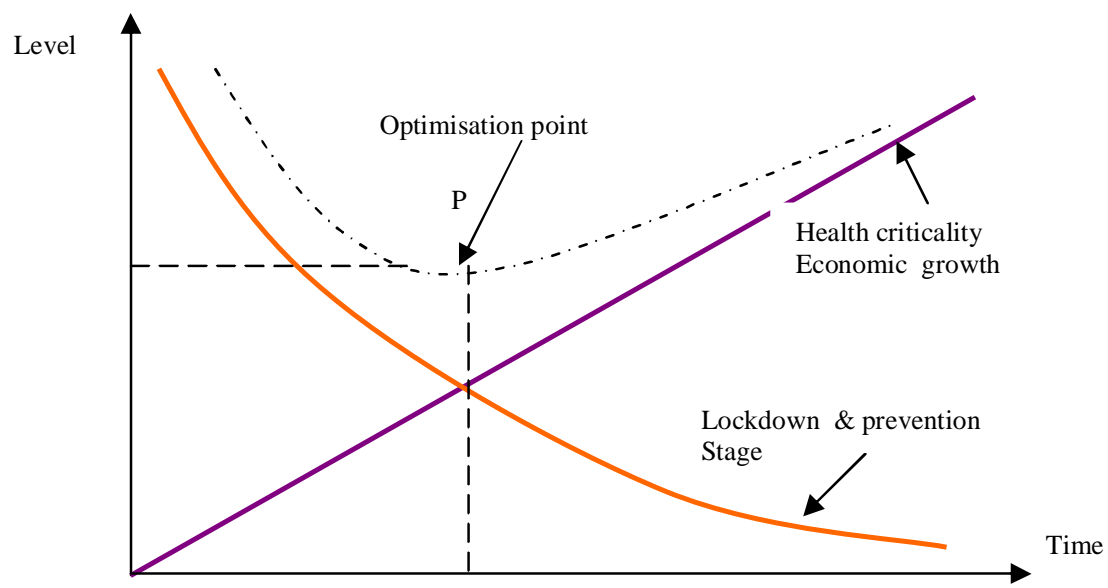

required as shown in Fig.15.

laboratory, medical imaging and disinfection.

Fig.14 Graphical presentation for an example of optimization.

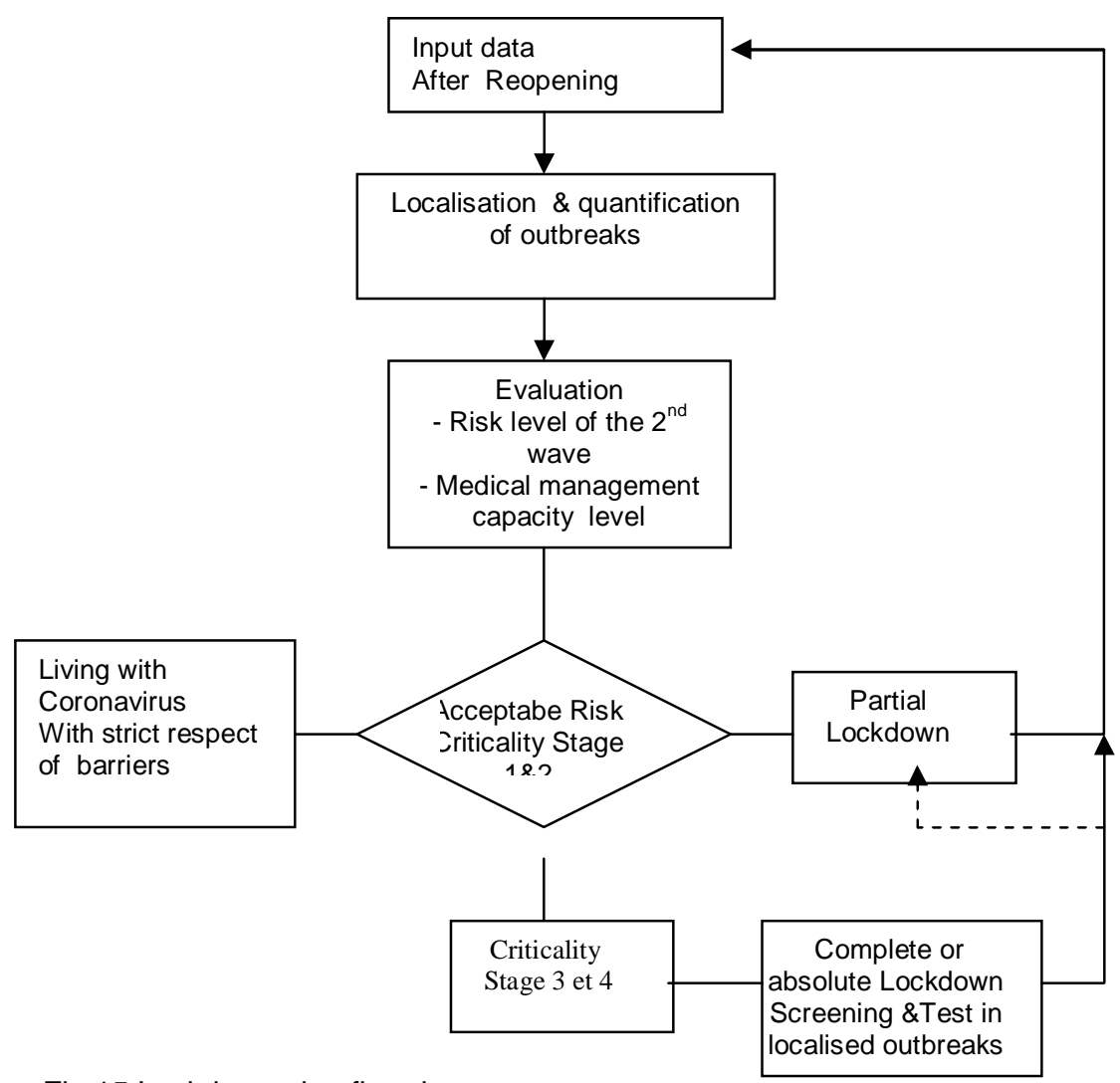

Fig.15 Lockdown plan flowchart.

For an effect of success of a mission such as the improvement in healthcare handling capacity, one carries out the identification of all the possible causes and factors, or secondary and up to the details to reach the cited desired objective using Ishikawa method.

- Recruiting and Training in reanimation service and the use of breathing devices, covid-19 test devices, medical imaging \& disinfection. 
- In view of the big efforts made, the medical staff are exhausted and under tremendous stress with fear of contamination. To continue their noble missions over time, they need more psychological support and medical facilitation means.

- Maintenance, availability and safety of breathing devices and their supply systems in electricity and oxygen.

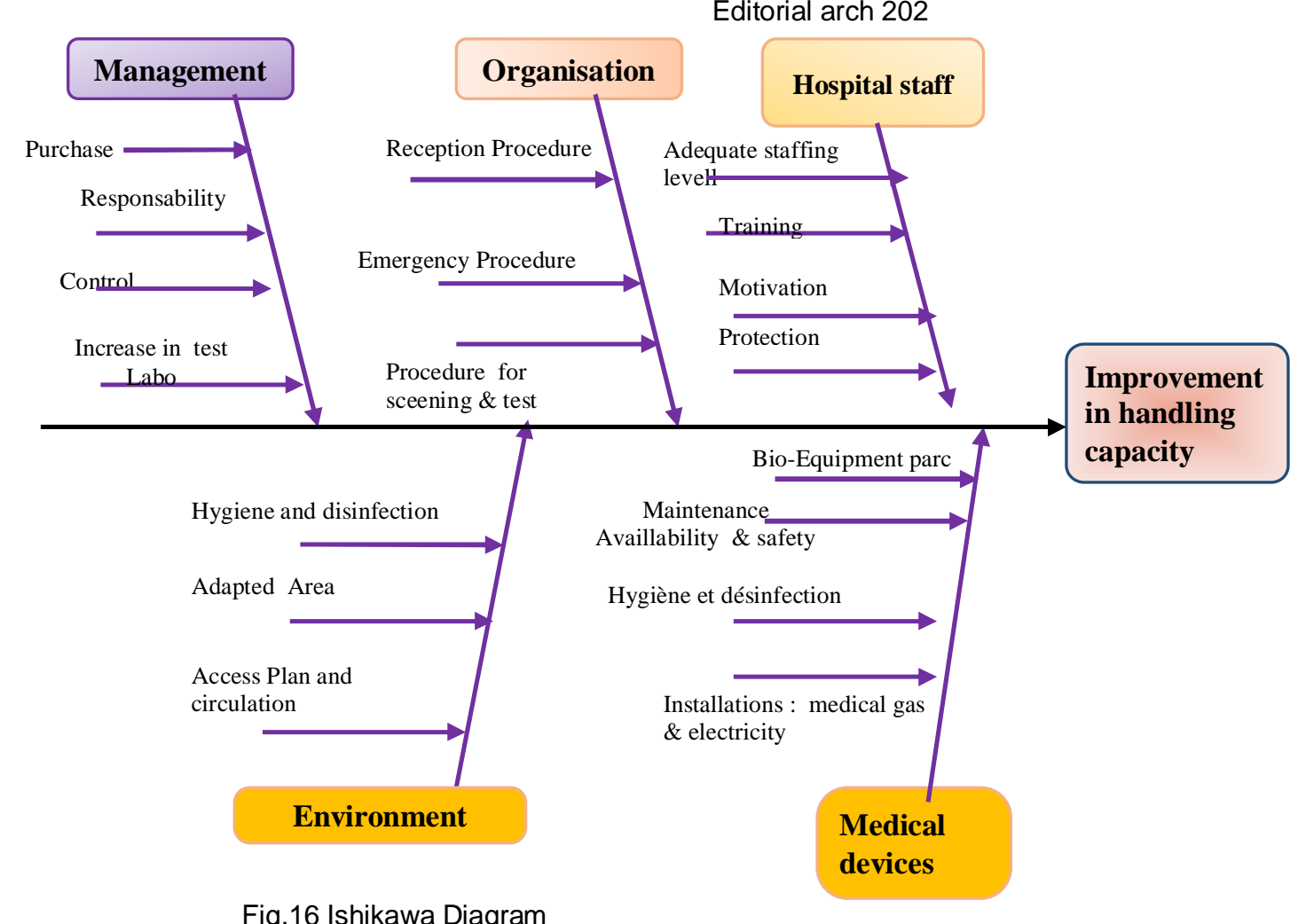

Our intention as experts in major risk management and biomedical engineering is to propose and adapt an approach commonly used in the high-risk industrial world and to contribute through the addition of scientific methods of analysis, risk management and decision-support tools to contribute and work towards the safeguarding of human lives in our country as well as around the world.

\section{References}

[1] Nichan Margossian, Risques et Accidents Industriels Majeurs: Caractéristiques, Réglementation, Prévention, Dunod, Paris, 2006.

[2] Cogan page, Paul Hopkin, Fundamentals of Risk Management, 4th Edition Institute of Risk Management (GBR), 2017.

[3] Wikipedia Pandemie de COVID-19 en Algérie .

[4] Public Health England as official guidance. Guidance for infection prevention and control in healthcare settings,

[5] Shanghai Institutes for International Studies. International Cooperation for the Coronavirus Combat: Results, Lessons, and Way Ahead,

[6] www.cdc.gov COVID19, Implementation of Mitigation Strategies for Communities with Local COVID-19 Transmission .

[7] Michael McAleer, Prevention Is Better Than the Cure: Risk Management of COVID-19 Journal of Risk and Financial Management Editorial arch 202 